\title{
TU/e EmonONEN

\section{Stark broadening experiments on a vacuum arc discharge in tin vapor}

Citation for published version (APA):

Kieft, E. R., Mullen, van der, J. J. A. M., Kroesen, G. M. W., Banine, V. Y., \& Koshelev, K. N. (2004). Stark broadening experiments on a vacuum arc discharge in tin vapor. Physical Review E - Statistical, Nonlinear, and Soft Matter Physics, 70(6), 66402-1. [66402]. https://doi.org/10.1103/PhysRevE.70.066402

DOI:

10.1103/PhysRevE.70.066402

Document status and date:

Published: 01/01/2004

\section{Document Version:}

Publisher's PDF, also known as Version of Record (includes final page, issue and volume numbers)

\section{Please check the document version of this publication:}

- A submitted manuscript is the version of the article upon submission and before peer-review. There can be important differences between the submitted version and the official published version of record. People interested in the research are advised to contact the author for the final version of the publication, or visit the $\mathrm{DOI}$ to the publisher's website.

- The final author version and the galley proof are versions of the publication after peer review.

- The final published version features the final layout of the paper including the volume, issue and page numbers.

Link to publication

\section{General rights}

Copyright and moral rights for the publications made accessible in the public portal are retained by the authors and/or other copyright owners and it is a condition of accessing publications that users recognise and abide by the legal requirements associated with these rights.

- Users may download and print one copy of any publication from the public portal for the purpose of private study or research.

- You may not further distribute the material or use it for any profit-making activity or commercial gain

- You may freely distribute the URL identifying the publication in the public portal.

If the publication is distributed under the terms of Article 25fa of the Dutch Copyright Act, indicated by the "Taverne" license above, please follow below link for the End User Agreement:

www.tue.nl/taverne

Take down policy

If you believe that this document breaches copyright please contact us at:

openaccess@tue.nl

providing details and we will investigate your claim. 


\title{
Stark broadening experiments on a vacuum arc discharge in tin vapor
}

\author{
E. R. Kieft, ${ }^{*}$ J. J. A. M. van der Mullen, ${ }^{\dagger}$ and G. M. W. Kroesen \\ Department of Applied Physics, Eindhoven University of Technology, P.O. Box 513, 5600 MB Eindhoven, The Netherlands \\ V. Banine \\ ASML Netherlands B.V., De Run 6501, 5504 DR Veldhoven, The Netherlands \\ K. N. Koshelev \\ ISAN, Troitsk, Moscow Region, 142092, Russia
}

(Received 22 July 2004; revised manuscript received 17 September 2004; published 10 December 2004)

\begin{abstract}
Pinched discharge plasmas in tin vapor are candidates for application in future semiconductor lithography tools. This paper presents time-resolved measurements of Stark broadened linewidths in a pulsed tin discharge. Stark broadening parameters have been determined for four lines of the Sn III spectrum in the range from 522 to $538 \mathrm{~nm}$, based on a cross-calibration to a Sn II line with a previously known Stark width. The influence of the electron temperature on the Stark widths is discussed. Results for the electron densities in the discharge are presented and compared to Thomson scattering results.
\end{abstract}

DOI: 10.1103/PhysRevE.70.066402

PACS number(s): $52.70 . \mathrm{Kz}, 32.60 .+\mathrm{i}, 52.25 . \mathrm{Os}, 52.80 .-\mathrm{s}$

\section{INTRODUCTION}

Discharge plasmas as powerful sources of extreme ultraviolet (euv) radiation have been or are being developed by a number of groups in the world; a selection can be found in [1-7]. An important potential application of such sources lies in the field of the next generation of semiconductor lithography, which requires radiation in a $2 \%$ wavelength band around $13.5 \mathrm{~nm}$. Traditionally, in many of these sources, xenon is applied as the working element, since it not only exhibits a transition array near the desired wavelength, but also, being a noble gas, it is not easily deposited on or chemically reacting with the surfaces of (multilayer mirror) optics. However, because of doubts whether the xenon-based sources will in the end be able to meet the power requirements from the industry, the center of attention has recently shifted towards the use of tin as an alternative working element [8]. Despite the obvious drawbacks (tin is a solid under ambient conditions), it is an interesting element because of the highly favorable shape of its spectrum in the euv range.

Since early 2003, a triggered vacuum arc in Sn vapor from the Russian Institute of Spectroscopy (ISAN) has been in operation in the ASML EUV laboratory in Veldhoven, The Netherlands. Several techniques have been applied for the characterization of this source, and, more generally, to identify the mechanisms and elementary processes that play important roles in this type of discharge. Among these techniques are time-resolved plasma imaging, euv spectrometry [9], and Thomson scattering [10]. Since detailed descriptions of the evolution of the plasma are already given in $[9,10]$, only the main points will be repeated here.

Before ignition of the discharge, a ring of capacitors that is connected to the electrodes is charged to a potential of

\footnotetext{
*Email address: e.r.kieft@tue.nl

${ }^{\dagger}$ Corresponding author. Email address: j.j.a.m.v.d.mullen@tue.nl
}

about $4 \mathrm{kV}$. The cathode, which serves as the bottom electrode, is covered with a thin layer of liquid tin and the discharge is ignited by the vaporization of a small amount of the tin (trigger phase). A current starts to flow once the partially ionized cloud of tin vapor has expanded towards the edge of the ring-shaped anode.

Due to the low inductance of the electrical circuit, the current rises very quickly to about $20 \mathrm{kA}$ (prepinch phase). The large electric current has two effects. First, it causes strong heating of the plasma and multiple ionization of tin up to at least $\mathrm{Sn}^{11+}$. The electron temperature can reach values up to around $30 \mathrm{eV}$ in this phase. Second, the electric current creates the "pinch" effect: the Lorentz forces acting on the charged particles in the plasma cause the plasma to collapse to a needlelike shape with radius of about $100 \mu \mathrm{m}$ on the axis of symmetry of the discharge (pinch phase). Measurements show that the strongest euv radiation is emitted in this phase.

Since the magnetic field confines the plasma only in the radial direction, the plasma can escape along the discharge axis and the pinch dies. Simultaneously, the electric current drops and the plasma starts to cool down and expand (decay phase). At the same time, the heat that was deposited into the tin layer on the cathode by the strong current during the prepinch and pinch phases causes additional evaporation of tin and the creation of a new, much cooler plasma.

For the recording of Thomson scattered spectra, a spectrometer was used that was optimized for wavelengths near the second harmonic at $532 \mathrm{~nm}$ of a pulsed neodymiumdoped yttrium aluminum garnet (Nd:YAG) laser. The arrangement was such that a horizontal cross section of the discharge could be imaged onto an intensified chargecoupled device (iCCD) camera, while the other dimension was reserved for wavelength information. A schematic image of the discharge electrodes and the imaged part of the plasma is shown in Fig. 1. 


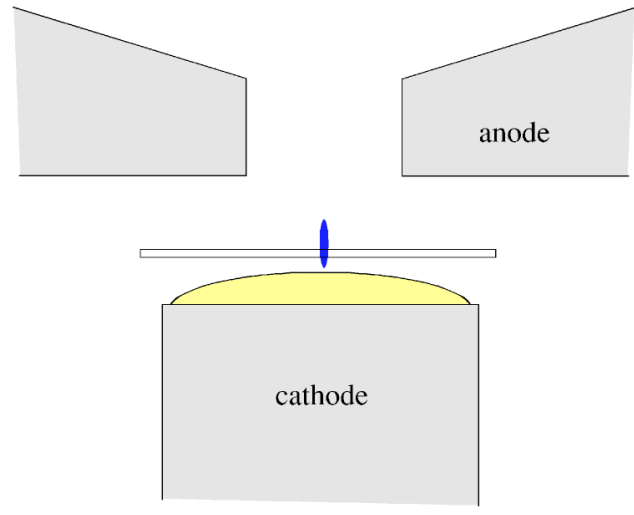

FIG. 1. A schematic cross section of the electrodes of the tin vapor discharge. The vertical elongated shape in the center represents the position of the pinch plasma. The horizontal rectangle shows the orientation and the approximate position of the plasma cross section as it was imaged by the spectrometer onto the iCCD camera.

During the Thomson scattering experiments, recordings were also made for the background radiation as emitted by the plasma itself, in wavelength ranges including the one of roughly $527-538 \mathrm{~nm}$. The presence of a few tin emission lines in this range with linewidths that were varying strongly during the evolution of the plasma led to a closer analysis of this part of the tin spectrum and the major line broadening mechanisms.

It was concluded that under the plasma conditions of the tin discharge, Stark broadening-due to collisions of the radiating atom with charged particles-is the dominant broadening mechanism. In principle, it is possible to derive electron densities from the Stark widths of broadened lines; this is a well-established spectroscopic technique $[11,12]$. Thus, measurement of the plasma emission would provide an additional method for determination of electron densities independent of the Thomson scattering technique. Using the same setup as the one for Thomson scattering provides the benefits of an accurate wavelength calibration and good time resolution due to the presence of an iCCD camera.

However, we found that the calculation of electron densities from the widths of the detected lines was not straightforward. Of the four tin lines in the $527-538 \mathrm{~nm}$ range, one was identified as a Sn II line, while the other three were found to be Sn III lines. Only for the Sn II line was the Stark broadening parameter known from the literature [13,14], but in certain spectra only the widths of the Sn III lines could be determined with sufficient accuracy. Further, we knew that for certain phases of the discharge, the electron temperature varies strongly, and is much higher than the temperature of $1.0 \times 10^{4} \mathrm{~K}$ and $1.16 \times 10^{4} \mathrm{~K}$, for which the broadening parameter was given.

In the following section, an approach will be presented for a cross-calibration of the Stark widths of the Sn III lines to the width of the $\mathrm{Sn}$ II line. Also, a theoretical justification is given for the interpretation of Stark broadening measurements at higher electron temperatures, and the importance of other possible broadening mechanisms is discussed. In Sec. III, a brief description of the setup and the experimental pro- cedure for the determination of the electron densities is given. In Sec. IV, the Stark broadening parameters for four Sn III spectral lines (three in the 527-538 $\mathrm{nm}$ range and one at a lower wavelength) are presented. Further, electron density values have been derived for all phases of the discharge except the pinch phase. These values are presented and compared to the Thomson scattering results for the prepinch phase. In the same section, the validity and accuracy of the results are discussed.

\section{STARK WIDTHS}

\section{A. Stark broadening parameters of the observed lines}

The following lines have been detected and identified as belonging to the $\mathrm{Sn}$ II and III spectra:

$$
\begin{gathered}
\text { Sn II } \quad 6 \mathrm{~d}^{2} \mathrm{D}_{3 / 2} \rightarrow 6 \mathrm{p}^{2} \mathrm{P}_{1 / 2}^{\mathrm{o}} \quad 533.39 \mathrm{~nm} \quad(\mathrm{a}), \\
\text { Sn III } \quad 6 \mathrm{p}^{3} \mathrm{P}_{1}^{\mathrm{o}} \rightarrow 5 \mathrm{~d}^{3} \mathrm{D}_{1} \quad 529.3 \mathrm{~nm} \quad \text { (b), } \\
6 \mathrm{p}^{3} \mathrm{P}_{1}^{\mathrm{o}} \rightarrow 5 \mathrm{~d}^{3} \mathrm{D}_{2} \quad 535.1 \mathrm{~nm} \quad \text { (c), } \\
6 \mathrm{p}^{3} \mathrm{P}_{0}^{\mathrm{o}} \rightarrow 5 \mathrm{~d}^{3} \mathrm{D}_{1} \quad 537.1 \mathrm{~nm} \quad \text { (d), } \\
6 \mathrm{p}^{1} \mathrm{P}_{1}^{\mathrm{o}} \rightarrow 6 \mathrm{~s}^{1} \mathrm{~S}_{0} \quad 522.6 \mathrm{~nm} \quad \text { (e). }
\end{gathered}
$$

In the following, these lines will also be referred to by their labels, (a)-(e), as given above. Lines (a)-(d) are in the $527-538 \mathrm{~nm}$ wavelength range, and could therefore all be recorded together in a single measurement. Line (e) is at a lower wavelength, and could therefore only be recorded together with line (b) in a single measurement over the 511.7-531.5 $\mathrm{nm}$ range.

Energy level and wavelength information for line (a) is given in [13,14]. References [13] and [14] also give Stark broadening values of $0.27 \pm 0.04 \mathrm{~nm}$ and $0.30 \pm 0.04 \mathrm{~nm}$, respectively, defined as the Lorentzian half-width at half maximum (HWHM) of the line at an electron density $n_{e}$ $=10^{23} \mathrm{~m}^{-3}$. The four Sn III lines (b)-(e) have been identified in the present study with the help of energy level information from [15].

Apart from these data, the literature information is, in view of our application, rather limited. For line (a), the broadening parameter has only been measured for electron temperatures of $1.16 \times 10^{4}$ and $1.0 \times 10^{4} \mathrm{~K}$, by Miller and Martínez, respectively, while the temperatures in the tin discharge under study can get up to tens of eV. Furthermore, for lines (b)-(e), to our knowledge no broadening information is available from the literature at all.

To solve the latter problem for lines (b)-(d), we have made a cross-calibration of their widths to the width of line (a), for those spectra for which both could be determined with sufficient accuracy. Nearly all of the data points are in the decay phase of the discharge. The widths of lines (b)-(d) were assumed to be directly proportional to the width of line (a), and for each line, the Stark broadening parameter was calculated from the proportionality constant as derived from the experimental data, and the Stark broadening parameter of 
line (a). For the latter, the average of the literature values was applied.

Line (e) has not been recorded simultaneously with line (a) in a single measurement, and therefore only an indirect cross-calibration of the Stark width using line (b) was possible. A Stark broadening parameter for this line has been calculated, but the indirect derivation of this value was not considered to be accurate enough to be useful for the subsequent determination of electron densities.

Although the Stark broadening is mainly determined by the electron density $n_{e}$ in the plasma, the electron temperature $T_{e}$ also has a certain influence. Before we can do an interpretation of the results obtained at higher electron temperatures, we need to evaluate the temperature dependency of the line broadening.

The electron impact broadening as presented by Griem [16] is given by the following expression:

$$
\begin{aligned}
w_{s e}= & 8\left(\frac{\pi}{3}\right)^{3 / 2} \frac{\hbar}{m a_{0}} n_{e}\left(\frac{E_{H}}{\kappa T_{e}}\right)^{1 / 2} \\
& \times\left[\left\langle i\left|r^{2}\right| i\right\rangle \bar{g}_{s e}\left(\xi_{i}\right)+\left\langle f\left|r^{2}\right| f\right\rangle \bar{g}_{s e}\left(\xi_{f}\right)\right],
\end{aligned}
$$

where $i$ and $f$ denote the initial and final states of the transition, respectively, $\xi_{i, f}=\frac{3}{2} \kappa T_{e} /\left|\Delta E_{i, f}\right|$ where $\Delta E_{i, f}$ are the energy differences to the nearest perturbing levels for those states, $\bar{g}_{s e}(\xi)$ is an effective Gaunt factor, and $w_{s e}$ is the HWHM of the Lorentz profile in frequency units. The other symbols have their usual meanings. $w_{s e}$ is proportional to the full width at half maximum (FWHM) line broadening $\Delta \lambda$ in wavelength units, through the expression $\Delta \lambda=w_{s e} \lambda^{2} /(\pi c)$.

According to Eq. (1), $w_{\text {se }}$ depends on the electron temperature both through an explicit $1 / \sqrt{T_{e}}$ dependency and through the effective Gaunt factors, which have $T_{e}$ in their arguments. It can be rewritten as

$$
w_{s e}=A \frac{\bar{g}_{s e}\left(\xi_{i}\right)}{\sqrt{\xi_{i}}}+B \frac{\bar{g}_{s e}\left(\xi_{f}\right)}{\sqrt{\xi_{f}}},
$$

where the prefactors $A$ and $B$ do not depend on $T_{e}$. In the approximation of Van Regemorter [17], the effective Gaunt factor $\bar{g}_{s e}(\xi)$ for positive ions tends to a constant value of 0.2 for $\xi \rightarrow 0$ and behaves roughly as $(\sqrt{3} / 2 \pi) \ln \xi$ for large values of $\xi$.

Now, the function $\bar{g}_{s e}(\xi) / \sqrt{\xi}$ exhibits only a very weak dependency on $\xi$ for a large range of values of $\xi$. This point is illustrated in Fig. 2. Therefore, as long as the $\xi_{i, f}$ values for both the initial and final states of a measured profile are not very far from those applicable to the calibration of the same line, an estimation of the electron density will be possible without prior detailed information on the electron temperature in the plasma. Also, Fig. 2 shows that $w_{s e}$ is constant within a factor 2 as long as both $\xi_{i, f}$ stay within a very wide range of roughly $1<\xi<200$. In the following, we will use the literature and calibration values of the Stark broadening parameters for the calculation of the electron densities, and check the validity of the method by evaluating the values of $\xi_{i, f}$ both for the calibration and for the electron density measurements.

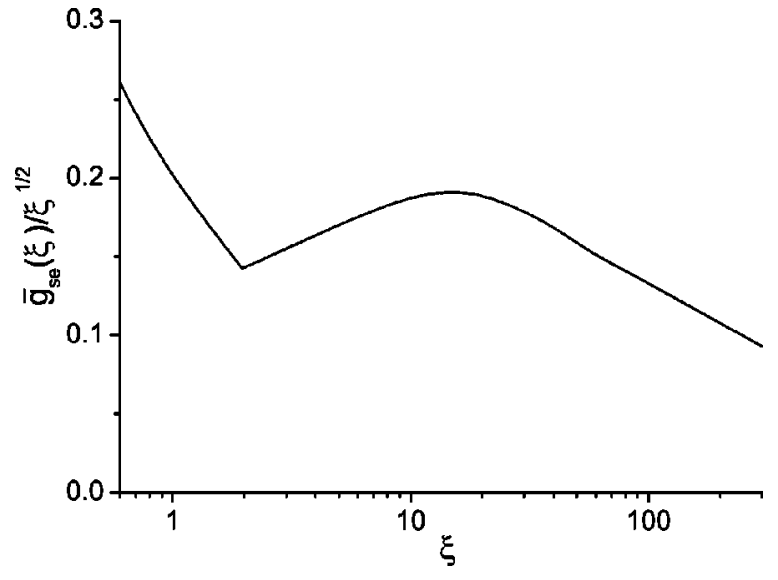

FIG. 2. The function $\bar{g}_{s e}(\xi) / \sqrt{\xi}$, with $\bar{g}_{s e}(\xi)$ as defined in [17]. The plot shows the very weak dependency of the function on $\xi$.

\section{B. Other broadening mechanisms}

Apart from Stark broadening, several other mechanisms can contribute to the broadening of spectral lines that are emitted by plasmas [11]. These include natural line broadening, Doppler broadening, and pressure broadening due to collisions with neutral atoms. However, given realistic plasma properties, natural line broadening is negligible compared to all other mechanisms. With an electron temperature of about $30 \mathrm{eV}$ or lower, Doppler broadening of tin lines near $532 \mathrm{~nm}$ will not exceed about $0.025 \mathrm{~nm}$. Further, since the interaction between charged particles is much stronger than the interaction with neutrals, and given the fact that the tin discharge has a considerable degree of ionization for all relevant phases, the pressure broadening due to collisions with neutral atoms will be much smaller than Stark broadening. Therefore, it can be concluded that Stark broadening will dominate over all of the broadening mechanisms mentioned above, provided that it is not very small compared to the width of the apparatus profile, which is about $0.16 \mathrm{~nm}$ FWHM, as discussed in Sec. III.

Macroscopic (external) electric and magnetic fields can also contribute to line broadening. An external electric field will cause Stark splitting of the emission lines. For large fields, the (energy) splitting of a level is of the order $S$ $=e a_{0} p^{2} \mathcal{E}$, where $\mathcal{E}$ represents the magnitude of the electric field, and the prefactor is a typical electric dipole matrix element with a perturbing level, where $a_{0}$ is the first Bohr radius and $p$ represents the principal quantum number of the upper level. However, as long as this value is small compared to the distance $\Delta$ to the closest perturbing level, only a small quadratic contribution remains (similar to the expression derived in [18] for the simplest case of only two strongly interacting levels),

$$
\Delta E \approx \frac{S^{2}}{\Delta}=\frac{\left(e a_{0} p^{2}\right)^{2}}{\Delta} \mathcal{E}^{2} .
$$

The magnitude of the electric field in the plasma will never exceed a value of about $1 \times 10^{6} \mathrm{~V} / \mathrm{m}$, which is near the values that we find when we divide the maximum potential of $4 \mathrm{kV}$ by the typical electrode distances. During the 


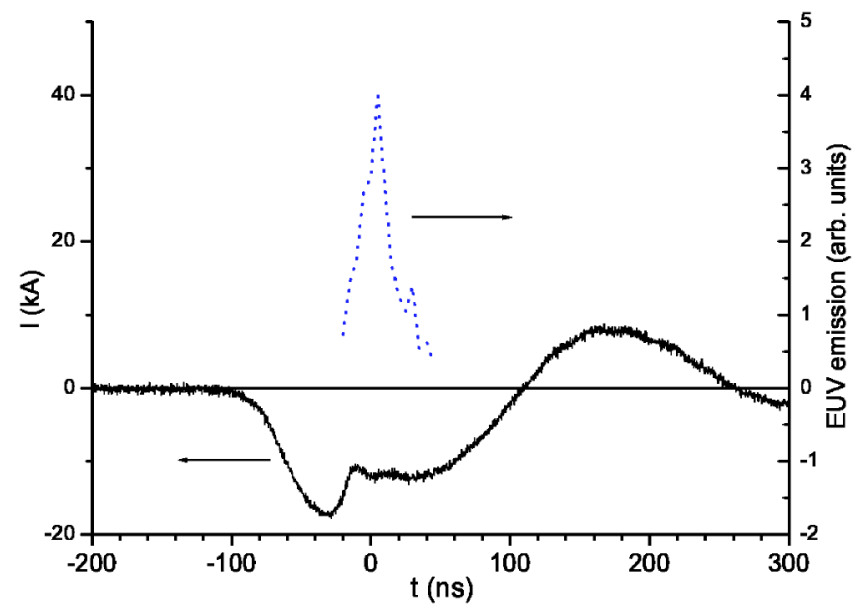

FIG. 3. Example of the discharge current (solid curve) and euv emission derived from plasma imaging experiments (dotted curve) during the discharge. The absolute value of the current is derived from the electrical circuit parameters, and is only approximate.

discharge, the typical electric fields will be even smaller since most of the potential drop between the electrodes will then be concentrated in the plasma sheaths. Now, for all lines, $S$ is of the order of $2 \mathrm{meV}$. The lower level of line (a) has an energy gap of $11 \mathrm{meV}$ to the nearest perturbing level; the other lines all have energy differences $\Delta=0.7 \mathrm{eV}$ or larger. This results in a line broadening of not more than $0.08 \mathrm{~nm}$ full width for line (a) and less than $1.2 \times 10^{-3} \mathrm{~nm}$ for the other lines. Therefore, only for line (a) could the electric field result in a detectable contribution to the broadening.

A similar argument is valid for the motional Stark effect, which results from the electric field that is sensed by a moving charged particle in the presence of a magnetic field. For the phases of the discharge for which measurements have been done, the magnetic field can reach a value of up to about $3.6 \mathrm{~T}$, as is discussed in detail below. For realistic plasma velocities of about $10^{4} \mathrm{~m} / \mathrm{s}$, the magnitude of the "motional electric field," $\mathcal{E}_{\text {mot }}=|\mathbf{v} \times \mathbf{B}| \approx 4 \times 10^{4} \mathrm{~V} / \mathrm{m}$, is even much smaller than the value of the electric field used for the estimations above.

Finally, the magnetic field can contribute to the line broadening directly through the Zeeman effect. Roughly, the line splitting in terms of energy will be of the order $\mu_{B} B$, where $\mu_{B}=e \hbar / 2 m_{e}=9.3 \times 10^{-24} \mathrm{~J} / \mathrm{T}$ is the Bohr magneton. Of all the phases for which measurements are available, the magnetic field will be the strongest in the prepinch phase. In this phase, the current increases from nearly zero to almost $20 \mathrm{kA}$ between about 90 and $30 \mathrm{~ns}$ before the pinch. An example of a current plot is shown in Fig. 3. Assuming a flat spatial current density distribution, and a constant plasma radius of $r \approx 0.7 \mathrm{~nm}$ before the start of the compression, the cross-section averaged magnetic-field strength increases from zero to about 3.6 T. This can lead to a full width line broadening of up to about $0.06 \mathrm{~nm}$ at $t=-30 \mathrm{~ns}$, which might give a significant contribution to the total broadening of the lines. The effects of the electric and magnetic fields on the results will be discussed in Sec. IV.

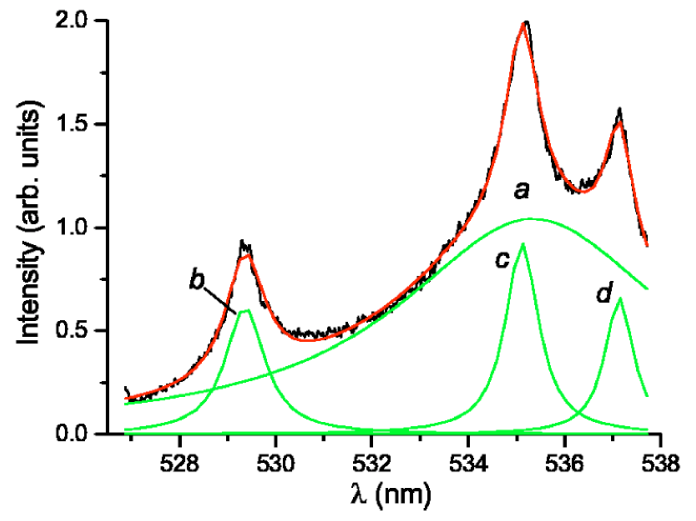

FIG. 4. Example of a plasma spectrum in the 527-538 nm wavelength range, recorded $120 \mathrm{~ns}$ after the pinch. Apart from the experimental data, also the individual line fit curves ( $a$, Sn II $533.39 \mathrm{~nm} ; \quad b$, Sn III $529.3 \mathrm{~nm} ; \quad c$, Sn III $535.1 \mathrm{~nm}$; $d$, Sn III $537.1 \mathrm{~nm}$ ) and their sum have been plotted. In this case, the Gaussian apparatus profile was negligible compared to the Lorentzian Stark contributions. A flat background contribution was subtracted from the experimental curve and has not been included in the graph.

\section{EXPERIMENT}

For all experiments, the triple-grating spectrometer (TGS) was applied as originally designed and built by Marco van de Sande [19] for application in Thomson scattering experiments, and slightly modified and used for work on the tin discharge [10]. Depending on the focal length of the last lens in the system, the effective spectral range of the system could be selected to be either about $10 \mathrm{~nm}$ or $20 \mathrm{~nm}$. The stray light reduction properties of the TGS were used only for simultaneous Thomson scattering measurements.

Stark widths have been determined both from the actual spectra that have been recorded as background in the Thomson scattering experiments, and from separately recorded plasma emission spectra, mainly for the trigger and decay phases of the discharge, in which TS was not feasible. The TS spectra are over the wavelength ranges of $511.7-531.5 \mathrm{~nm}$ and $532.6-552.9 \mathrm{~nm}$, while the separately recorded spectra cover the 526.9-537.7 nm range. In both cases, only the vertically polarized signal has been considered. In the former case, only one or three of the four lines (a)-(d) could be used, respectively, for determination of the electron density. In the latter case, lines (a)-(d) were all available.

The camera's optical gating time was always 5 ns. The camera was synchronized to the ignition of the discharge, and a delay generator was used to vary the timing of the measurements relative to the evolution of the discharge. The time resolution of the measurements was about $10 \mathrm{~ns}$, being limited both by the optical gating time of the camera and the pulse-to-pulse jitter in the timing of the discharge evolution, which also contributed about $5 \mathrm{~ns}$. Both single-shot and multiple-shot averaged experiments have been performed.

The width of the apparatus profile of the TGS in the spectral direction has been determined using the widths of the Sn III lines from the outer regions of the plasma during the decay phase, where the electron densities are very low and 
TABLE I. Full widths at half maximum of lines (a) to (d) for measurements in the 527-538 nm range. The last column gives the electron density values derived using the calibration data as described in the text.

\begin{tabular}{|c|c|c|c|c|c|}
\hline $\mathrm{t}(\mathrm{ns})$ & $\Delta \lambda_{a}(\mathrm{~nm})$ & $\Delta \lambda_{b}(\mathrm{~nm})$ & $\Delta \lambda_{c}(\mathrm{~nm})$ & $\Delta \lambda_{d}(\mathrm{~nm})$ & $n_{e}\left(10^{23} \mathrm{~m}^{-3}\right)$ \\
\hline-270 & 0.82 & & & & 1.5 \\
\hline-240 & 0.85 & & & & 1.5 \\
\hline-210 & 0.69 & & & & 1.2 \\
\hline-180 & 0.77 & & & & 1.4 \\
\hline-150 & 0.58 & & & & 1.0 \\
\hline-120 & 0.48 & 0.08 & & & 0.88 \\
\hline-90 & 0.60 & 0.08 & 0.07 & 0.04 & 0.91 \\
\hline-80 & 0.98 & & & & 1.7 \\
\hline-70 & 1.06 & & & & 1.9 \\
\hline-60 & & 0.25 & 0.17 & 0.16 & 2.7 \\
\hline-50 & & 0.21 & 0.18 & 0.16 & 2.7 \\
\hline-40 & & 0.37 & 0.26 & 0.32 & 4.5 \\
\hline 60 & 6.95 & 0.90 & 0.70 & 0.68 & 11 \\
\hline 70 & 7.88 & 1.05 & 0.94 & 0.83 & 14 \\
\hline 80 & 7.00 & 1.09 & 0.94 & 0.76 & 13 \\
\hline 90 & 6.94 & 1.00 & 0.92 & 0.78 & 13 \\
\hline 100 & 6.65 & 0.98 & 0.89 & 0.85 & 13 \\
\hline 110 & 6.72 & 1.19 & 0.89 & 0.95 & 14 \\
\hline 120 & 6.75 & 1.30 & 0.66 & 0.73 & 12 \\
\hline 150 & 5.34 & 1.04 & 0.58 & 0.80 & 11 \\
\hline 180 & 3.82 & 0.62 & 0.33 & 0.38 & 6.4 \\
\hline 210 & 3.36 & 0.34 & 0.24 & 0.28 & 4.6 \\
\hline 240 & 2.66 & & & & 4.8 \\
\hline 270 & 2.24 & & & & 4.0 \\
\hline 300 & 1.52 & & & & 2.7 \\
\hline 330 & 1.25 & & & & 2.2 \\
\hline 360 & 1.06 & & & & 1.9 \\
\hline 390 & 0.91 & & & & 1.6 \\
\hline 420 & 0.66 & & & & 1.2 \\
\hline 450 & 0.60 & & & & 1.1 \\
\hline 480 & 0.46 & & & & 0.81 \\
\hline 510 & 0.46 & & & & 0.82 \\
\hline 540 & 0.31 & & & & 0.56 \\
\hline 570 & 0.33 & & & & 0.59 \\
\hline 600 & 0.29 & & & & 0.52 \\
\hline 700 & 0.23 & & & & 0.41 \\
\hline 800 & 0.19 & & & & 0.34 \\
\hline 900 & 0.19 & & & & 0.34 \\
\hline 1100 & 0.09 & & & & 0.16 \\
\hline 1300 & 0.12 & & & & 0.21 \\
\hline 1500 & 0.04 & & & & 0.073 \\
\hline
\end{tabular}

hence the contribution of the apparatus profile to the total measured widths of the lines could be expected to be dominant. The apparatus profile has been found to have a FWHM of $0.16 \mathrm{~nm}$.

Subsequently, the line profiles have been fitted to a Voigt profile, with a fixed Gaussian contribution to take the apparatus profile into account, and a Lorentzian contribution with a variable width. The Lorentzian contribution was fully ascribed to Stark broadening. The spectra over the $527-538 \mathrm{~nm}$ range for which the widths of lines (a)-(d) could all be determined with sufficient accuracy were used for the determination of Stark broadening parameters, as discussed in the previous section.

After this, for every spectrum the electron density was 
TABLE II. Stark broadening parameters $\Delta \lambda$ for four lines of the Sn III spectrum, given in the form of half-widths at half maximum of the Lorentzian profiles in wavelength units for $n_{e}=10^{23} \mathrm{~m}^{-3}$ and $T_{e}=1 \mathrm{eV}$. Also given are the estimated relative errors in these numbers, as well as theoretical broadening parameters $\Delta \lambda_{\text {se }}$, derived using the semiempirical formula by Griem [16].

\begin{tabular}{lcccc}
\hline \hline Line & & $\Delta \lambda(\mathrm{nm})$ & Rel. error & $\Delta \lambda_{\text {se }}(\mathrm{nm})$ \\
\hline (b) & $6 \mathrm{p}^{3} \mathrm{P}_{1}^{\mathrm{o}} \rightarrow 5 \mathrm{~d}^{3} \mathrm{D}_{1}$ & 0.043 & $30 \%$ & 0.030 \\
(c) & $6 \mathrm{p}^{3} \mathrm{P}_{1}^{\mathrm{o}} \rightarrow 5 \mathrm{~d}^{3} \mathrm{D}_{2}$ & 0.034 & $30 \%$ & 0.030 \\
(d) & $6 \mathrm{p}^{3} \mathrm{P}_{0}^{\mathrm{o}} \rightarrow 5 \mathrm{~d}^{3} \mathrm{D}_{1}$ & 0.032 & $30 \%$ & 0.030 \\
(e) & $6 \mathrm{p}^{1} \mathrm{P}_{1}^{\mathrm{o}} \rightarrow 6 \mathrm{~s}^{1} \mathrm{~S}_{0}$ & 0.061 & $50 \%$ & 0.036 \\
\hline \hline
\end{tabular}

calculated as a simple average of the electron density values derived from the individual lines for which the broadening could be determined with sufficient accuracy.

In principle, radially resolved spectra of the axisymmetric discharge could have been obtained by Abel inversion at each wavelength of the entire spectrum. However, such a procedure would have been extremely laborious and therefore we have chosen to limit ourselves to fitting of only the central spectrum from the lateral profile, thereby effectively obtaining an electron density estimate for the entire cross section of the discharge. Since the highest density regions of the plasma will be emitting the strongest radiation and therefore contribute to the measured spectrum the most, we assume that this effective electron density will be close to the peak value of the density profile, to within about $25 \%$.

\section{RESULTS AND DISCUSSION}

An example of the plasma emission spectrum in the $527-538 \mathrm{~nm}$ range is shown in Fig. 4. In Table I, FWHM values are given for the linewidths in the same spectral range for different times in the evolution of the discharge.

The Stark broadening parameters for lines (b) to (d), derived from cross-calibration to the widths of line (a) for those entries in Table I for which all widths are present, are given in Table II. Also, the Stark parameter for line (e), derived from comparison of its width with that of line (b), is included.

In Sec. II, some limitations were given for the temperature ranges of the Stark results. However, the parameters in Table II were determined almost exclusively from measurements in the decay phase, which is a recombining plasma and therefore necessarily has a low electron temperature. From the model of Colombant and Tonon [20], it follows that in a stationary plasma the electron temperature must be between 1 and $3 \mathrm{eV}$ when both $\mathrm{Sn}^{+}$and $\mathrm{Sn}^{2+}$ are abundant. For a recombining plasma, the electron temperature will be even lower. As a result, the actual temperature will not be far from those used in $[13,14]$, and the Stark broadening parameter of line (a) will be close to the literature values. Similarly, the Stark broadening parameters in Table II can be interpreted as being valid for an electron temperature of $1 \mathrm{eV}$. We estimate the errors in the Stark broadening parameters due to uncertainties in the electron temperature to be not more than about $\pm 20 \%$.

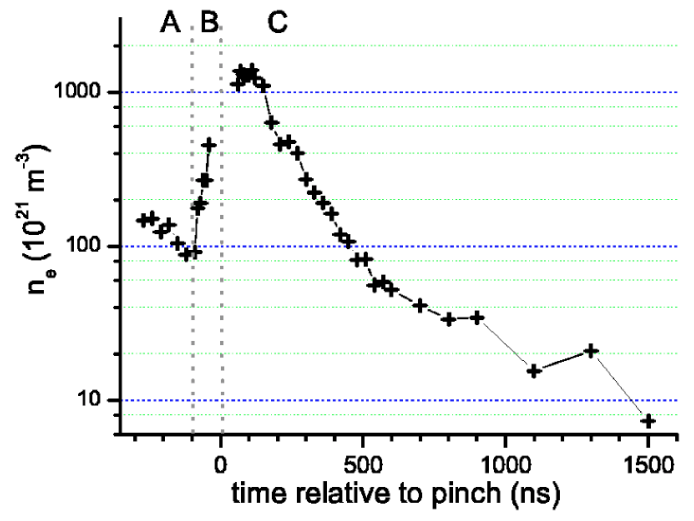

FIG. 5. Electron densities as calculated from Stark broadening. The time scale has been subdivided into three parts: A, trigger plasma; B, prepinch phase; C, decay phase. No Stark broadening data are available for the pinch phase (around $0 \mathrm{~ns}$ on the time scale).

Other possible error sources include the uncertainties in the literature data $[13,14]$ on the broadening of line $(a)$, the spatial gradients in the electron density, and macroscopic electric and magnetic fields in the plasma. However, since the calculation of Stark parameters is based on a comparison between linewidths, the errors due to the spatial variations in the electron density will partially cancel out. Further, electric and magnetic fields do not play an important role in the decay phase of the discharge, as will be discussed below. Since more data are available for lines (b) to (d) than for line (e), and the Stark parameter for line (e) has been derived only from an indirect measurement, the error estimate for line (e) is larger than for the other three.

When we compare the experimentally determined Stark widths in Table II with the semiempirical formula of Griem (1), using his approximation

$$
\left\langle x\left|r^{2}\right| x\right\rangle=\frac{p_{x}^{2}}{2(z+1)^{2}}\left[5 p_{x}^{2}+1-3 l_{x}\left(l_{x}+1\right)\right] a_{0}^{2},
$$

where $x$ represents either $i$ or $f, p_{x}$ is the effective principal quantum number, $l_{x}$ is the orbital quantum number, and $z$ is the charge number of the level under consideration, we see that all results match to within a factor 2. A more detailed, quantum-mechanical calculation of the Stark broadening parameters was beyond the scope of this work.

In Fig. 5, the electron densities in the plasma, as derived from the 527-538 $\mathrm{nm}$ spectra, have been plotted versus time. The durations of the trigger plasma, prepinch phase, and decay phases of the main discharge are indicated for reference. Electron densities from the Stark broadening measurements in the prepinch phase are shown together with electron densities and temperatures from Thomson scattering experiments in Fig. 6. Results from multiple measurement series of Stark broadening, including those from the background spectra of actual Thomson scattering experiments, have been included in this figure to give an impression of the spread in the results. The figure shows that there is excellent agreement in magnitude and trend for both sources of electron density information. 

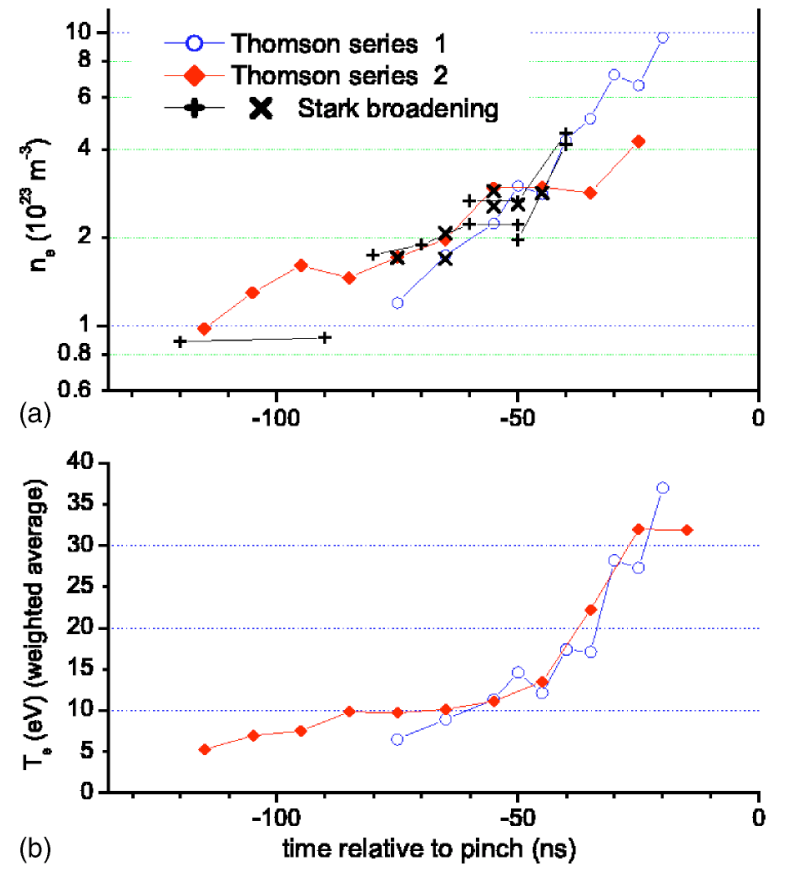

FIG. 6. (a) Electron densities as derived from multiple series of Stark broadening data, and maximum fitted electron densities per profile from Thomson scattering, plotted as a function of time before the pinch. (b) Profile-averaged electron temperatures from Thomson scattering, weighted by the electron density.

Again, the temperature limitations as discussed in Sec. II should be checked. From energy level data in [15], it follows that for line (a), $\Delta E_{i, f}$ are rather small with values of only about 0.1 and $0.01 \mathrm{eV}$, respectively. This leads to large values of $\xi$ for the temperatures that can typically be found in the tin discharge plasma. However, the width of line (a) was used almost exclusively for measurements in the trigger and decay phases, which are both recombining plasmas. Following the discussion above, we can conclude that the electron temperatures in those phases will not be far from $1 \mathrm{eV}$, so that the Stark broadening parameters from the literature can be applied.

On the other hand, lines (b), (c), and (d) also play a role in the determination of the electron densities in the ionizing prepinch plasma. For these measurements, the electron temperature has values up to $16 \mathrm{eV}$, as can be derived from Fig. 6 . For these lines, the tables in [15] give values for $\Delta E_{i, f}$ of 0.7 and $2.0 \mathrm{eV}$, respectively, and hence the energy differences to the nearest perturbing levels are much larger than for line (a). Now, $\xi_{i, f}$ vary from 2 and 0.75 , respectively, in the decay phase (assuming $T_{e} \approx 1 \mathrm{eV}$ ) to up to 34 and 12 in the prepinch phase for the initial and final states, respectively (with $T_{e}=16 \mathrm{eV}$ ). For this range of $\xi$ values, Fig. 2 shows that $\bar{g}_{s e}(\xi) / \sqrt{\xi}$ is only a very weak function of temperature, and hence also for the $\mathrm{Sn}$ III lines the condition derived in Sec. II is met.

Finally, the possible contributions of the electric and magnetic fields to the line broadening were mentioned in Sec.
II B. For the electric field, it was derived there that it could give an additional broadening to line (a) of not more than $0.08 \mathrm{~nm}$. As Table I shows, this contribution would be significant only after $t=500 \mathrm{~ns}$. However, this is already far into the decay phase of the discharge and the capacitors will already have been drained of their charge almost completely, so that no large electric field is present anymore. Comparison of the estimated magnetic-field contributions to the actually measured linewidths for the measurements in the prepinch phase show that the presence of the magnetic field might lead to an overestimation of the electron density by about $20 \%$. In the other phases, the magnetic field is much smaller and/or the linewidths are much larger, so that the magnetic field does not lead to significant additional broadening.

Taking all sources of error into account, it seems reasonable to assume an error margin of about $\pm 50 \%$ for the measured electron densities in the discharge.

\section{CONCLUSIONS}

In this work, measurements of electron densities have been presented using the Stark broadening of emission lines of singly and doubly ionized tin in a vacuum arc pulsed discharge. The Stark broadening parameter was known from the literature for only one line in the part of the spectrum under study. From our measurements, we have been able to derive Stark broadening parameters for four new Sn III lines with up to $\pm 30 \%$ accuracy. Since a part of the measurements was done simultaneously with Thomson scattering experiments, the results from both techniques could be compared. Even when taking into account that a certain part of the tin line broadening in the prepinch phase is caused by the presence of a magnetic field, a good agreement both in the absolute values and in the trend of the data is found. The agreement in the absolute values can be considered as a confirmation of the validity of the cross-calibration of the Stark broadening parameters.

The measurements show that the electron density in the prepinch phase, which is of the order $10^{23} \mathrm{~m}^{-3}$ before the start of the discharge current, increases to several times its earlier value in only about $50 \mathrm{~ns}$ time, thereby confirming the trend in the Thomson scattering results. Also, a decrease of the density from about $10^{24} \mathrm{~m}^{-3}$ to below $10^{22} \mathrm{~m}^{-3}$ in a longer time span of about $1.5 \mu$ s during the decay of the plasma has been shown.

In the discharge under study, the electron temperature varied over a large range, and in some cases was far from the values for which the Stark broadening parameter of the Sn II line was known from the literature. Despite these unfavorable circumstances, we have shown both in theory and in experiment that the Stark broadening technique can provide fairly accurate electron density information in the $\mathrm{Sn}$ euv discharge over a range of more than two decades. This is due to the fact that the Sn III lines are about an order of magnitude narrower than that of the Sn II line. Stark broadening can therefore be a useful additional tool for characterization of this type of plasma. 
[1] M. A. Klosner and W. T. Silfvast, Opt. Lett. 23, 1609 (1998).

[2] S. R. Mohanty, C. Cachoncinlle, C. Fleurier, E. Robert, J.-M. Pouvesle, R. Viladrosa, and R. Dussart, Microelectron. Eng. 61-62, 179 (2002).

[3] M. McGeoch, Appl. Opt. 37, 1651 (1998).

[4] V. M. Borisov, I. Ahmad, S. Goetze, A. S. Ivanov, O. B. Khristoforov, J. Kleinschmidt, V. Korobotchko, J. Ringling, G. Schriever, U. Stamm, and A. Y. Vinokhodov, Proc. SPIE 4688, 626 (2002).

[5] W. N. Partlo, I. V. Fomenkov, R. M. Ness, R. I. Oliver, S. T. Melnychuk, and J. E. Rauch, Proc. SPIE 4343, 232 (2001).

[6] M. W. McGeoch and C. T. Pike, Proc. SPIE 5037, 141 (2003).

[7] K. Bergmann, G. Schriever, O. Rosier, M. Müller, W. Neff, and R. Lebert, Appl. Opt. 38, 5413 (1999).

[8] Proceedings of the EUV Source Workshop, International SEMATECH, Antwerp, Belgium, 2003, edited by V. Bakshi (unpublished).

[9] E. R. Kieft, J. J. A. M. van der Mullen, G. M. W. Kroesen, V. Banine and K. N. Koshelev (unpublished).

[10] E. R. Kieft, J. J. A. M. van der Mullen, G. M. W. Kroesen, and
V. Banine, Phys. Rev. E 70, 056413 (2004).

[11] H. R. Griem, Principles of Plasma Spectroscopy (Cambridge University Press, Cambridge, 1997).

[12] N. Konjević, Phys. Rep. 316, 339 (1999).

[13] M. H. Miller, R. A. Roig, and R. D. Bengtson, Phys. Rev. A 20, 499 (1979).

[14] Be. Martínez and F. Blanco, J. Phys. B 32, 241 (1999).

[15] C. E. Moore, Atomic Energy Levels: As Derived from the Analysis of Optical Spectra (U.S. National Bureau of Standards, Washington, D.C., 1971).

[16] H. R. Griem, Phys. Rev. 165, 258 (1968).

[17] H. van Regemorter, Astrophys. J. 136, 906 (1962).

[18] I. I. Sobelman, Introduction to the Theory of Atomic Spectra (Pergamon, Oxford, 1972).

[19] M. J. van de Sande, Ph.D. thesis, Eindhoven University of Technology, 2002, http://alexandria.tue.nl/extra2/ 200210414.pdf

[20] D. Colombant and G. F. Tonon, J. Appl. Phys. 44, 3524 (1973). 